\title{
Artificial Halo Orbits for Low-Thrust Propulsion Spacecraft
}

\author{
Shahid Baig • Colin R. McInnes
}

Received: date / Accepted: date

\begin{abstract}
We consider periodic halo orbits about artificial equilibrium points near to the Lagrange points $L_{1}$ and $L_{2}$ in the circular restricted three body problem, where the third body is a low-thrust propulsion spacecraft in the Sun-Earth system. Although such halo orbits about artificial equilibrium points can be generated using a solar sail, there are points inside $L_{1}$ and beyond $L_{2}$ where a solar sail cannot be placed, so lowthrust, such as solar electric propulsion, is the only option to generate artificial halo orbits around points inaccessible to a solar sail. Analytical and numerical halo orbits for such low-thrust propulsion systems are obtained by using the Lindstedt Poincaré and differential corrector method respectively. Both the period and minimum amplitude of halo orbits about artificial equilibrium points inside $L_{1}$ decreases with an increase in low-thrust acceleration. The halo orbits about artificial equilibrium points beyond $L_{2}$ in contrast show an increase in period with an increase in low-thrust acceleration. However, the minimum amplitude first increases and then decreases after the thrust acceleration exceeds $0.415 \mathrm{~mm} / \mathrm{s}^{2}$. Using a continuation method, we also find stable artificial halo orbits which can be sustained for long integration times and require a reasonably small low-thrust acceleration $0.0593 \mathrm{~mm} / \mathrm{s}^{2}$.
\end{abstract}

Keywords Restricted three body problem - halo orbits · low-thrust propulsion · continuation method

\section{Introduction}

It is well-known that the circular restricted three-body problem (CRTBP) has five natural equilibrium points. Three of them are on the axis joining the primaries (collinear

Shahid Baig, PhD Candidate

Department of Mechanical Engineering, University of Strathclyde, Glasgow, G1 1XJ, Scotland, UK.

E-mail: shahid.baig@strath.ac.uk

Colin R. McInnes, Professor

Department of Mechanical Engineering, University of Strathclyde, Glasgow, G1 1XJ, Scotland, UK.

E-mail: colin.mcinnes@strath.ac.uk 
Lagrange points) and two are on the vertices of equilateral triangles joining the primaries (equilateral Lagrange points). At Lagrange points the gravitational forces of the two primaries and the centrifugal force on a spacecraft in a rotating frame are balanced. Artificial equilibrium points (AEPs) other than Lagrange points can be generated by using a constant continuous acceleration from a low-thrust propulsion system such as a solar sail or solar electric propulsion system.

Around the collinear Lagrange points, 'classical' halo orbits have been extensively studied, for example Farquhar and Kamel [1], Breakwell and Brown [2], Richardson [3], Howell [4], Thurman and Worfolk [5]. Notably, Richardson [3,6] used the method of Lindstedt Poincaré to obtain a third-order analytical approximation of periodic halo orbits (unstable) in a simple, high-precision and straightforward manner. Stable halo orbits were found by Breakwell and Brown around $L_{2}$ in the Earth-Moon system [2], and later on by Howell [4] for a wide range of mass ratios around all three collinear Lagrange points in an extensive numerical study.

McInnes et al. [7] show continuous surfaces of AEPs can be generated in the CRTBP for a solar sail low-thrust propulsion system, but only in certain allowed regions. These AEPs are characterized by the sail lightness number and sail orientation. The linearized eigenvalue spectrum around AEPs contains at least one centre, so linear periodic orbits can be generated or the Lindstedt Poincaré method can be applied. McInnes [8] and Baoyin and McInnes [9], describe halo orbits around AEPs on the line joining the two primaries in the solar-sail three body problem. However, McInnes [8] describes stable regions of halo orbits around unstable AEPs, when the amplitude of the halo orbit becomes large. Waters and McInnes [10] generate unstable 'artificial' halo orbits in the solar-sail CRTBP about AEPs, which are high above the ecliptic plane. However, for a solar sail, all of these 'artificial' halo orbits around AEPs, in and above the eclliptic plane, are in the allowed/accessible volume of space.

Morimoto et al. [11] find AEPs in the CRTBP for a solar electric or nuclear electric low-thrust propulsion system. These AEPs are characterized by the low-thrust acceleration magnitude and thrust orientation. In particular, marginally stable regions in addition to unstable regions of AEPs are found that differ from the solar sail problem which has only unstable regions of AEPs. Morimoto et al. [12] also find resonant periodic orbits with a constant, continuous acceleration at linear order around the marginally stable AEPs along the axis joining the primary bodies. In this paper, we extend this analysis of low-thrust periodic orbit at nonlinear order using the Lindstedt Poincaré method about unstable AEPs on the line joining the two primaries. Furthermore, we show the feasibility of halo orbits about AEPs where a solar sail cannot generate periodic orbits because of the requirement that the sail acceleration cannot be directed towards the Sun i.e., for AEPs inside $L_{1}$ and beyond $L_{2}$. The AEPs are chosen near to the natural Lagrange points $L_{1}$ and $L_{2}$ to limit the power and thrust level from the low-thrust propulsion system. We also show the existence of stable halo orbits around unstable AEPs beyond $L_{2}$, using the orbit half-period as a continuation parameter which results better convergence accuracy. Furthermore, spacecraft on stable orbits are found to require a reasonably small low-thrust acceleration pointing towards the Sun.

In the next two sections we outline the equations of motion and periodic orbits at linear order for a low-thrust system in the CRTBP as given by Morimoto et al. [12] but in compact manner, as that information is needed for analysis of the nonlinear system. However, we have shown that the system admits a constant of motion since the equations of motion are explicitly independent of time. 


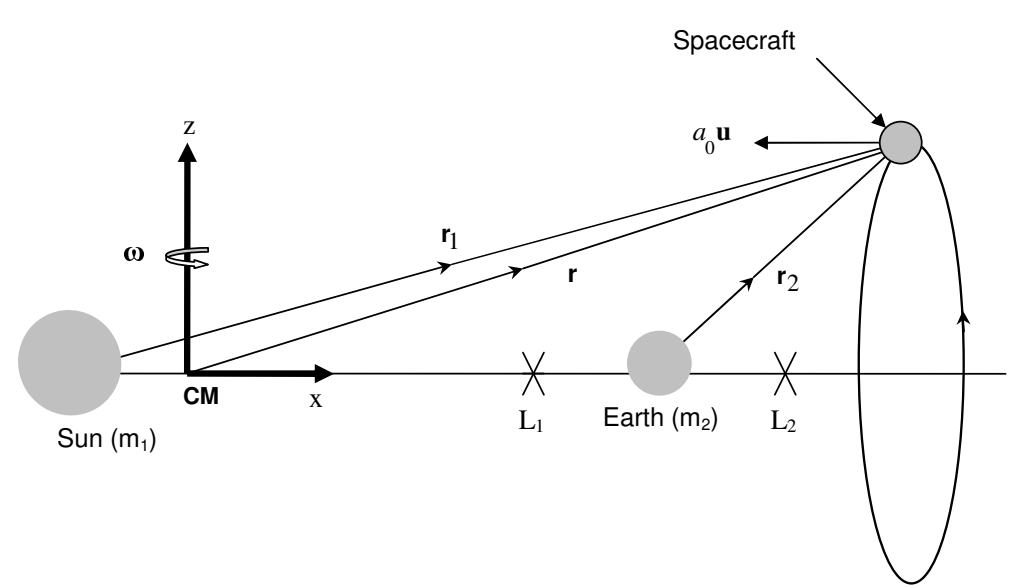

Fig. 1 Definition of coordinate system and low-thrust spacecraft in a periodic halo orbit about an artificial equilibrium point beyond $L_{2}$.

\section{Equations of Motion}

The CRTBP is a dynamical model that describes the motion of an infinitesimal mass, a spacecraft under the gravitational influence of two massive bodies in circular motion. Consider a synodic coodinate frame i.e., co-rotating with the two primary masses $m_{1}$ and $m_{2}$ at constant angular velocity $\boldsymbol{\omega}$ with origin at their center of mass, as shown in Fig. 1. The $\mathrm{x}$-axis points along the Sun-Earth line, the $\mathrm{z}$-axis is the axis of rotation and the y-axis completes the right-handed coordinate system. The system is made nondimensional by taking the units of length, mass and time such that distance between the primaries, the product of gravitational constant $G$ and sum of the masses of the primaries, and the period of the primaries is 1,1 and $2 \pi$ respectively. By defining $\mu=\frac{m_{2}}{m_{1}+m_{2}}, m_{1}$ is located at $(-\mu, 0,0)$ and $m_{2}$ is located at $(1-\mu, 0,0)$ with respect to centre of mass. If we denote $\mathbf{r}=\left[\begin{array}{lll}x & y & z\end{array}\right]^{T}$ as the position vector of the low-thrust spacecraft relative to the centre of mass, then the position vector of the spacecraft with respect to the primaries $m_{1}$ and $m_{2}$ is given by

$$
\mathbf{r}_{1}=\left[\begin{array}{lll}
x+\mu & y & z
\end{array}\right]^{T}, \quad \mathbf{r}_{2}=[x-(1-\mu) \quad y \quad z]^{T}
$$

The nondimensional equation of motion of a low-thrust spacecraft in the rotating frame of reference is given by

$$
\ddot{\mathbf{r}}+2 \boldsymbol{\omega} \times \dot{\mathbf{r}}=\nabla V+\boldsymbol{a}_{0} \equiv \boldsymbol{F}
$$

where $V$ is the effective potential given by

$$
V=\left(\frac{1-\mu}{r_{1}}+\frac{\mu}{r_{2}}\right)+\frac{1}{2}\left(x^{2}+y^{2}\right)
$$

The vector $\boldsymbol{a}_{0}$ is the acceleration due to the low-thrust propulsion system. At an equilibrium point $\ddot{\mathbf{r}}$ and $\dot{\mathbf{r}}$ vanish, so an equilibrium point is a zero of $\boldsymbol{F}$ i.e., $\boldsymbol{F}\left(\mathbf{r}_{0}\right)=\mathbf{0}$. Thus, a nonequilibrium point $\mathbf{r}_{0}$ in the rotating frame is changed into an artificial equilibrium point with low-thrust acceleration vector $\boldsymbol{a}_{0}$ satisfying the following condition

$$
-\nabla V=\boldsymbol{a}_{0}\left(\mathbf{r}_{0}\right)=a_{0} \mathbf{u}
$$


where magnitude and direction of low-thrust acceleration is given by

$$
\begin{aligned}
& a_{0}=|\nabla V| \\
& \mathbf{u}=-\frac{\nabla V}{|\nabla V|}
\end{aligned}
$$

Taking the dot product on both sides of Eq. (1) by $\dot{\mathbf{r}}=\boldsymbol{v}$, we get

$$
\boldsymbol{v} \cdot \dot{\boldsymbol{v}}+2 \boldsymbol{v} \cdot(\boldsymbol{\omega} \times \boldsymbol{v})-\boldsymbol{v} \cdot \boldsymbol{a}_{0}=\boldsymbol{v} \cdot \nabla V=\frac{d \mathbf{r}}{d t} \cdot \frac{\partial V}{\partial \mathbf{r}}
$$

or

$$
\frac{d\left[\frac{1}{2} \boldsymbol{v}^{T} \boldsymbol{v}-\boldsymbol{a}_{0}^{T} \mathbf{r}\right]}{d t}=\frac{d V}{d t}
$$

So we have the Jacobi constant for the low-thrust system given by

$$
C(\mathbf{r}, \boldsymbol{v})=\frac{1}{2} \boldsymbol{v}^{T} \boldsymbol{v}-\boldsymbol{a}_{0}^{T} \mathbf{r}-V(\mathbf{r})
$$

For the correct initial conditions, the spacecraft will move on a periodic orbit around an artificial equilibrium point $\mathbf{r}_{0}$ with constant continuous acceleration $\boldsymbol{a}_{0}$ satisfying Eq. (3), and having the constant of motion $C$.

The classical case (with no propulsion) is Hamiltonian and time independent, so we have an energy integral of motion and this energy is defined by Eq. (4) with $\boldsymbol{a}_{0}=$ $(0,0,0)$. At the Lagrange points $L_{1}$ and $L_{2}$ of the Sun-Earth system, the energies of the spacecraft at rest are -1.500448970 and -1.500446943 respectively.

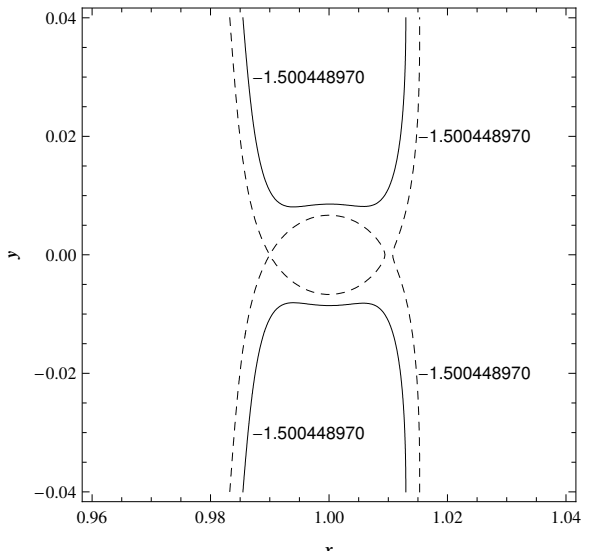

(a)

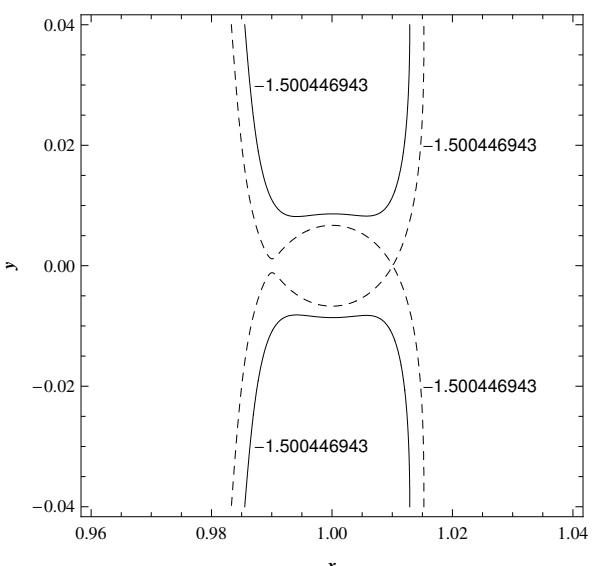

(b)

Fig. 2 Zero velocity curves in the Sun-Earth system for (a) energy values of the $L_{1}$ and (b) energy values of the $L_{2}$ point . For classical case $\boldsymbol{a}_{0}=(0,0,0)$ (dashed contour lines) and for low-thrust system $\boldsymbol{a}_{0}=(0.0001,0,0)$ (solid contour lines)

Fig. 2 shows that classical case contours are closed for energy values at $L_{1}$ and $L_{2}$ points (see dashed contour lines). However, in case of a low-thrust system for $\boldsymbol{a}_{0}=$ $(0.0001,0,0)$ and contours with the same energy values (see solid contour lines), the contours at $L_{1}$ and $L_{2}$ points are opened, permitting the spacecraft to escape from the Sun-Earth system. 


\section{Linearized System}

A linear system $\delta \dot{\mathbf{X}}=A \delta \mathbf{X}$ in the vicinity of an equilibrium point $\mathbf{r}_{0}$ is obtained from the nonlinear system Eq. (1) by using the transformation $\mathbf{r}=\mathbf{r}_{0}+\delta \mathbf{r}$, where $\mathbf{r}_{0}=\left(x_{0}, 0,0\right), \delta \mathbf{r}=(\delta x, \delta y, \delta z)^{T}$ and $\delta \mathbf{X}=(\delta \mathbf{r}, \delta \dot{\mathbf{r}})^{T}$. We assume the attitude of the low-thrust system $\mathbf{u}$ is not perturbed so as to restrict the stability analysis in the sense of Lyapunov, furthermore $\boldsymbol{a}_{0}$ is fixed w.r.t. perturbation $\delta \mathbf{r}$ i.e., $\frac{\partial \boldsymbol{a}_{0}}{\partial \mathbf{r}}=0$. Then the Jacobian matrix $A$ is given by

$$
A=\left(\begin{array}{cc}
0 & I \\
M & \Omega
\end{array}\right)
$$

where $\mathrm{I}$ is the unity matrix. Moreover,

$$
M=\left.\frac{\partial \nabla V}{\partial \mathbf{r}}\right|_{\mathbf{r}_{0}}=\left(\begin{array}{lll}
a & 0 & 0 \\
0 & b & 0 \\
0 & 0 & e
\end{array}\right), \Omega=\left(\begin{array}{ccc}
0 & 2 & 0 \\
-2 & 0 & 0 \\
0 & 0 & 0
\end{array}\right)
$$

and

$$
a=2 c+1, b=1-c, e=-c
$$

with

$$
c\left(x_{0}, \mu\right)=\frac{\mu}{\left|x_{0}+\mu-1\right|^{3}}+\frac{1-\mu}{\left|x_{0}+\mu\right|^{3}}>0
$$

as $\mu>0$ and $1-\mu>0$. In Eq. (5), the $z$ equation is decoupled from $x, y$ equations for the AEP $\mathbf{r}_{0}$ chosen on the $x$-axis (or in the $x-y$ ecliptic plane), so the out of ecliptic plane equation of motion is given by

$$
\delta \ddot{z}+c \delta z=0
$$

which has a simple harmonic solution $\delta z=A_{z} \sin \left(w_{z} t+\phi_{z}\right)$, where $w_{z}=\sqrt{c}$. The characteristic polynomial for the $x, y$ linearized Eq. (5) rewritten in matrix form

$$
\left(\begin{array}{c}
\delta \dot{x} \\
\delta \dot{y} \\
\delta \ddot{x} \\
\delta \ddot{y}
\end{array}\right)=\left(\begin{array}{cccc}
0 & 0 & 1 & 0 \\
0 & 0 & 0 & 1 \\
a & 0 & 0 & 2 \\
0 & b & -2 & 0
\end{array}\right)\left(\begin{array}{c}
\delta x \\
\delta y \\
\delta \dot{x} \\
\delta \dot{y}
\end{array}\right)
$$

is given by

$$
p(\lambda)=\lambda^{4}+(2-c) \lambda^{2}+\left(1+c-2 c^{2}\right)
$$

By letting $\alpha=\lambda^{2}$, then the roots of $p(\alpha)=0$ are as follows

$$
\alpha_{1}=\frac{c-2+\sqrt{9 c^{2}-8 c}}{2}, \quad \alpha_{2}=\frac{c-2-\sqrt{9 c^{2}-8 c}}{2}
$$

We are now looking for AEPs where $c\left(x_{0}, \mu\right)>1$ (the unstable region) and not the marginally stable region (where $8 / 9 \leq c\left(x_{0}, \mu\right)<1$ ) [11], then $9 c^{2}-8 c>(c-2)^{2}$. From Eq. (7), then $\alpha_{1}>0$ and $\alpha_{2}<0$. So the eigenvalues spectrum of Eq. (6) consists of a saddle and center $\left\{ \pm i \lambda_{1}, \pm \lambda_{r}\right\}$, where $\lambda_{1}=w_{x y}=\sqrt{-\alpha_{2}}$ and $\lambda_{r}=\sqrt{\alpha_{1}}$. 
Let $\boldsymbol{u}_{1}+i \boldsymbol{w}_{1}$ be an eigenvector of the linearized Eq. (6) corresponding to eigenvalue $i \lambda_{1}$ and let $\boldsymbol{v}_{1}$ and $\boldsymbol{v}_{2}$ be the eigenvectors corresponding to eigenvalues $+\lambda_{r}$ and $-\lambda_{r}$. Then, the generalized solution of Eq. (6) is [13]

$$
\begin{gathered}
\left(\begin{array}{c}
\delta x \\
\delta y \\
\delta \dot{x} \\
\delta \dot{y}
\end{array}\right)=\cos \left(w_{x y} t\right)\left[A \boldsymbol{u}_{1}+B \boldsymbol{w}_{1}\right]+\sin \left(w_{x y} t\right)\left[B \boldsymbol{u}_{1}-A \boldsymbol{w}_{1}\right] \\
+C e^{\lambda_{r} t} \boldsymbol{v}_{1}+D e^{-\lambda_{r} t} \boldsymbol{v}_{2}
\end{gathered}
$$

where

$$
\boldsymbol{u}_{1}=\left(0,\left(a+w_{x y}^{2}\right), 2 w_{x y}^{2}, 0\right)^{T}, \boldsymbol{w}_{1}=\left(-2 w_{x y}, 0,0, w_{x y}\left(a+w_{x y}^{2}\right)\right)^{T}
$$

We set $C=0$ and $D=0$ to switch off the real modes to get bounded solutions for $\delta x$ and $\delta y$. Finally, the three-dimensional bounded solution to the linear problem Eq. (5) can be written as

$$
\begin{gathered}
\delta x=-A_{x} \cos \left(w_{x y} t+\phi_{x y}\right), \quad \delta y=k A_{x} \sin \left(w_{x y} t+\phi_{x y}\right) \\
\delta z=A_{z} \sin \left(w_{z} t+\phi_{z}\right)
\end{gathered}
$$

with $k=\frac{a+w_{x y}^{2}}{2 w_{x y}}$. For the AEPs in this paper, the ratio of in-plane $w_{x y}$ and out of frequencies $w_{z}$ is not a rational number, so a quasi-periodic Lissajous trajectory can be obtained as shown in Fig. 3 .
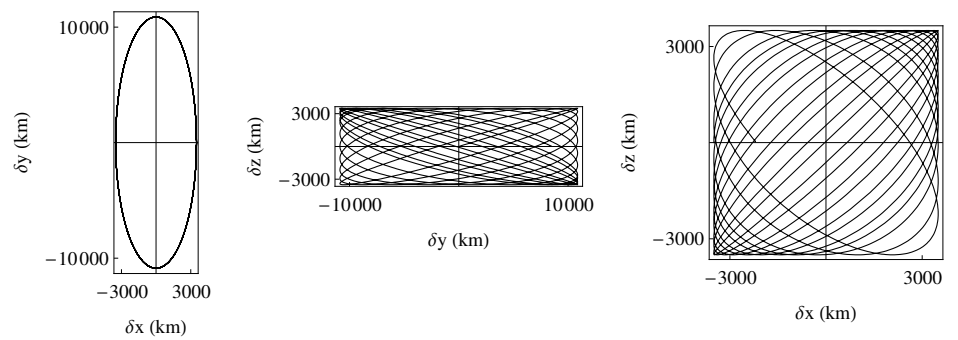

Fig. 3 Lissajous trajectory at AEP $\mathbf{r}_{0}=\left[\begin{array}{lll}1.02 & 0 & 0\end{array}\right]^{T}$ (beyond $L_{2}$ ) in the Sun-Earth system. $A_{x}=A_{z}=2.3396 \times 10^{-5}(3500 \mathrm{~km})$ and $\phi_{x y}=\phi_{z}=0$ are chosen for illustration purpose. The AEP needs $\boldsymbol{a}_{0}=(-0.0512,0,0)$.

\section{Nonlinear Approximations}

The Lindstedt Poincaré method is used to find periodic approximations to the equations of motion Eq. (1). In the CRTBP literature, Lindstedt Poincaré $[3,5]$ is used quite extensively to force the two linear frequencies $\left(w_{x y}\right.$ and $\left.w_{z}\right)$ to be equal by contributing nonlinear terms if the amplitudes $A_{x}$ and $A_{z}$ of the linear solution are large enough. The method is based on the assumption that if the nonlinearities are small, then the frequency of the periodic solution to the nonlinear system is a perturbation of the 
frequency of a periodic solution to the linear system. Therefore, the nonlinearity alters the frequency from $w_{x y}$ to $w_{x y} w$. where

$$
w=1+\epsilon w_{1}+\epsilon^{2} w_{2}+\ldots
$$

This frequency correction allows us to remove secular terms through determination of $w_{i}$ during the development of the approximate periodic solution about AEPs.

A Taylor series expansion of $\boldsymbol{F}$ to third-order [14] about AEP $\mathbf{r}_{0}$ is found by making the transformation $\mathbf{r} \rightarrow \mathbf{r}_{0}+\delta \mathbf{r}$, so we have the system of nonlinear equations

$$
\begin{gathered}
\delta \ddot{\mathbf{r}}+2 \boldsymbol{\omega} \times \delta \dot{\mathbf{r}}=\boldsymbol{F}\left(\mathbf{r}_{0}\right) \\
+\left.\left(\delta \mathbf{r} \cdot\left[\frac{\partial}{\partial \mathbf{r}}\right]^{T}\right) \nabla V\right|_{\mathbf{r}=\mathbf{r}_{0}}+\left.\frac{1}{2 !}\left(\delta \mathbf{r} \cdot\left[\frac{\partial}{\partial \mathbf{r}}\right]^{T}\right)^{2} \nabla V\right|_{\mathbf{r}=\mathbf{r}_{0}} \\
+\left.\frac{1}{3 !}\left(\delta \mathbf{r} \cdot\left[\frac{\partial}{\partial \mathbf{r}}\right]^{T}\right)^{3} \nabla V\right|_{\mathbf{r}=\mathbf{r}_{0}}+O\left(\delta \mathbf{r}^{4}\right)
\end{gathered}
$$

where we have assumed that $\frac{\partial \boldsymbol{a}_{0}}{\partial \mathbf{r}}, \frac{\partial^{2} \boldsymbol{a}_{0}}{\partial \mathbf{r}^{2}}$ etc., are all zero. So in component form the equations of motion through third-order are given by

$$
\begin{aligned}
\delta \ddot{x}-2 \delta \dot{y}-(2 c+1) \delta x= & 3 C\left(2 \delta x^{2}-\delta y^{2}-\delta z^{2}\right) \\
& +4 D \delta x\left(2 \delta x^{2}-3 \delta y^{2}-3 \delta z^{2}\right)+O\left(\delta \mathbf{r}^{4}\right) \\
\delta \ddot{y}+2 \delta \dot{x}+(c-1) \delta y= & -6 C \delta x \delta y \\
& -3 D \delta y\left(4 \delta x^{2}-\delta y^{2}-\delta z^{2}\right)+O\left(\delta \mathbf{r}^{4}\right) \\
\delta \ddot{z}+w_{x y}^{2} \delta z= & -6 C \delta x \delta z \\
& -3 D \delta z\left(4 \delta x^{2}-\delta y^{2}-\delta z^{2}\right)+O\left(\delta \mathbf{r}^{4}\right)+\Delta \delta z
\end{aligned}
$$

where $C=\frac{\left.V_{x x x}\right|_{\mathbf{r}=\mathbf{r}_{0}}}{12}$ and $D=\frac{\left.V_{x x x x}\right|_{\mathbf{r}=\mathbf{r}_{0}}}{48}$ are evaluated at the AEP $\mathbf{r}_{0}=\left(x_{0}, 0,0\right)$. Note that the term $\Delta=w_{x y}^{2}-c=w_{x y}^{2}-w_{z}^{2}=O\left(A_{z}^{2}\right)=O\left(\epsilon^{2}\right)$ is added in the righthand-side of the $z$-equation to force a periodic orbit at linear order with frequency $w_{x y}$ (this periodic solution is given in Eq. (9) with $w_{z}$ replaced by $w_{x y}$ and acts as a first approximation).

The following relations exist to switch off the secular term which appear as a result of successive approximation in the inhomogeneous part of the system of equations of order $O\left(\epsilon^{2}\right)$ and $O\left(\epsilon^{3}\right)$.

$$
\begin{gathered}
w_{1}=0, \quad w_{2}=s_{1} A_{x}^{2}+s_{2} A_{z}^{2} \\
l_{1} A_{x}^{2}+l_{2} A_{z}^{2}+\Delta=0, \quad \phi_{z}=\phi_{x y}+n \pi / 2 \quad n=1,3
\end{gathered}
$$

The expressions for $s_{i}, l_{i}$ are given in Ref. [5]. The closed orbit corresponding to these constraints is a halo orbit. Northern halo orbits, whose maximum out-of-plane component is above the ecliptic plane, are obtained corresponding to the solution $n=1$ and $n=3$ about AEPs near $L_{1}$ and $L_{2}$ respectively. The period of the orbit can be found from the amplitude-frequency relation $T=2 \pi / w_{x y} w$, where $w=1+s_{1} A_{x}^{2}+s_{2} A_{z}^{2}$. The minimum in-plane amplitude $A_{x \min }=\sqrt{\left|\Delta / l_{1}\right|}$ required to have a halo orbit can be derived from the above amplitude-constraint relation by substituting $A_{z}=0$. The 
complete third-order successive approximation solution of Eq. (11) using the Lindstedt Poincaré method is given by [5]

$$
\begin{aligned}
\delta x(t)= & -A_{x} \cos \tau_{1}+a_{21} A_{x}^{2}+a_{22} A_{z}^{2}+\left(a_{23} A_{x}^{2}+\zeta a_{24} A_{z}^{2}\right) \cos 2 \tau_{1} \\
& +\left(a_{31} A_{x}^{3}+\zeta a_{32} A_{x} A_{z}^{2}\right) \cos 3 \tau_{1} \\
\delta y(t)= & k A_{x} \sin \tau_{1}+\left(b_{21} A_{x}^{2}+\zeta b_{22} A_{z}^{2}\right) \sin 2 \tau_{1}+\left(b_{31} A_{x}^{3}+\zeta b_{32} A_{x} A_{z}^{2}\right) \sin 3 \tau_{1} \\
& +\left(b_{33} A_{x}^{3}+b_{34} A_{x} A_{z}^{2}+\zeta b_{35} A_{x} A_{z}^{2}\right) \sin \tau_{1} \\
\delta z(t)= & (-1)^{(n-1) / 2} A_{z} \cos \tau_{1}+(-1)^{(n-1) / 2} d_{21} A_{x} A_{z}\left(\cos 2 \tau_{1}-3\right) \\
& +(-1)^{(n-1) / 2}\left(d_{32} A_{z} A_{x}^{2}-d_{31} A_{z}^{3}\right) \cos 3 \tau_{1}
\end{aligned}
$$

where $\zeta=(-1)^{n}$ and $\tau_{1}=w_{x y} w t+\phi$. The constants $s_{i}, l_{i}, a_{i j}, b_{i j}$ and $d_{i j}$ involve $C$, $D, k$ and $w_{x y}$ which in turn ultimately depend on $\mathbf{r}_{0}$ and $\mu$. Note that the numerical values of these constants will be different from that given in $[3,5]$ due to the different scaled system chosen in nondimensionalization (see Sect. 2). In Eq. (12) the expression for $\delta y(t)$ also contains the third-order correction to the amplitude of $\sin \tau_{1}$ by Thurman and Worfolk [5] in Richardson's original solution [3]. This correction allows faster convergence of the differential corrector algorithm. Fig. 4 shows that the magni-

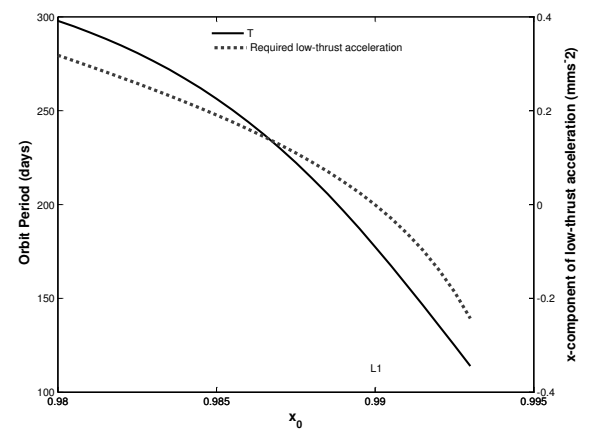

(a)

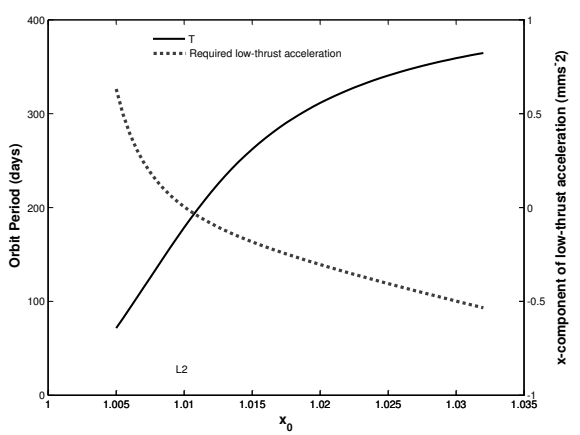

(b)

Fig. 4 Period of artificial halo orbit vs AEPs selected at $x_{0}$ near to (a) $L_{1}$ and (b) $L_{2}$ points in the Sun-Earth system. The dotted curve shows the low-thrust acceleration required at $x_{0}$ to create AEPs. $\boldsymbol{a}_{0}=0$ at $L_{1}$ and $L_{2}$.

tude of the low-thrust acceleration $\boldsymbol{a}_{0}$ is zero at $L_{1}$ and $L_{2}$ and increases to convert a nonequilibrium point at $x_{0}$ away from $L_{1}$ and $L_{2}$ into an equilibrium point. Artificial $L_{1}$ and $L_{2}$ points are chosen that require a maximum $a_{0} \approx 0.05\left(0.296 \mathrm{mms}^{-2}\right)$ and $a_{0} \approx 0.1\left(0.593 \mathrm{mms}^{-2}\right)$, which corresponds to a thrust of $150 \mathrm{mN}$ and $300 \mathrm{mN}$ for a $500 \mathrm{~kg}$ spacecraft. For points inside $L_{1}$ and beyond $L_{2}$, the direction of $\boldsymbol{a}_{0}$ is sunward, so these periodic artificial halo with a given $z$-amplitude cannot be generated with a solar sail.

Fig. 4 also shows that period $T=\frac{2 \pi}{\left(1+w_{2}\right) w_{x y}}$ of these artificial halo orbits follow the inverse behaviour of the zero order frequency $w_{x y}$ (see Fig. 5 ). Fig. 5 shows that the second order frequency correction to the zero order is very small, or roughly speaking a maximum $<3.5 \%$ frequency correction to the zero order solution (as $w_{1}=0$ ) for $A_{z}=$ $8.3557 \times 10^{-4}(125000 \mathrm{~km})$. Fig. 5 also shows that the correction can increase/decrease 


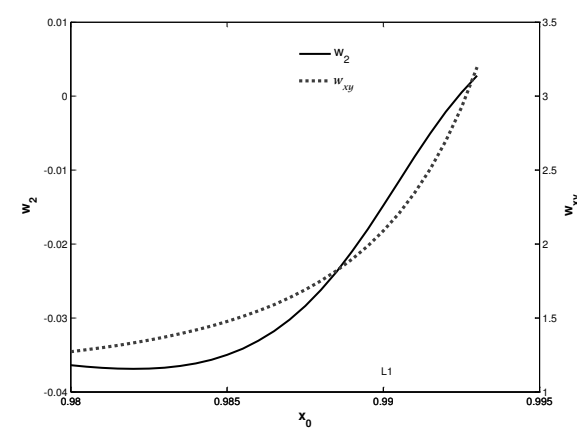

(a)

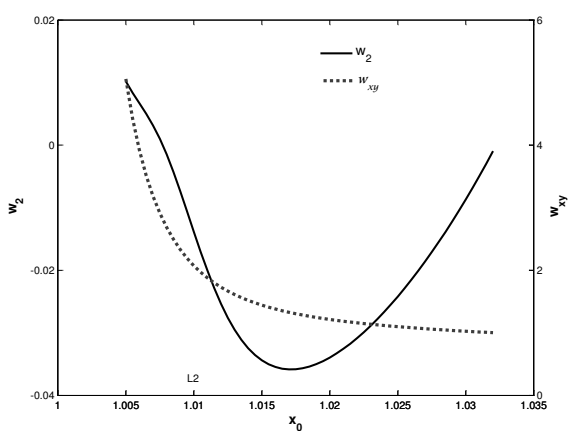

(b)

Fig. 5 Zero and second order frequency adjustment $\left(w_{2}\right)$ vs AEPs selected at $x_{0}$ near to (a) $L_{1}$ and (b) $L_{2}$ points in the Sun-Earth system.

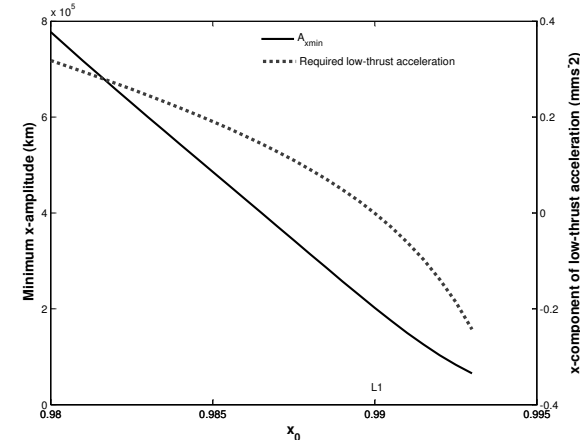

(a)

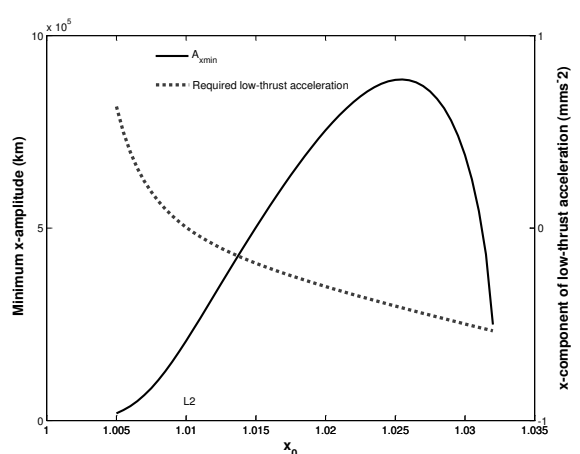

(b)

Fig. 6 The minimum x-amplitude to have artificial halo orbits vs AEPs selected at $x_{0}$ near to (a) $L_{1}$ and (b) $L_{2}$ points.

the period. Fig. 6 shows that the minimum amplitude $A_{x \min }=\sqrt{\left|\frac{\Delta}{l_{1}}\right|}$ beyond artificial $L_{2}$ points first increases then decreases when $a_{0}$ exceeds $\approx 0.07\left(0.415 \mathrm{mms}^{-2}\right)$, as at this point the rate at which $\sqrt{\Delta}$ decreases becomes more than the rate at which $\frac{1}{\sqrt{\left|l_{1}\right|}}$ increases. Although in Figs. (4-5) $A_{z}$ is chosen $125000 \mathrm{~km}$, the effect of $A_{z}$ on the second order frequency correction $w_{2}$, and so period $T$ is relatively small.

\section{Differential Correction and Low-Thrust Halo orbits}

We can use the initial guess from Lindstedt Poincaré analysis to integrate the full nonlinear system of equations Eq. (1) along with the constant low-thrust acceleration $\boldsymbol{a}_{0}$ to generate periodic orbits around AEP $\mathbf{r}_{0}$. The trajectory will start looking like a periodic orbit but will not close as Lindstedt Poincaré method generate (periodic) solutions that are approximations to the periodic solutions of the full nonlinear equations of motion. 


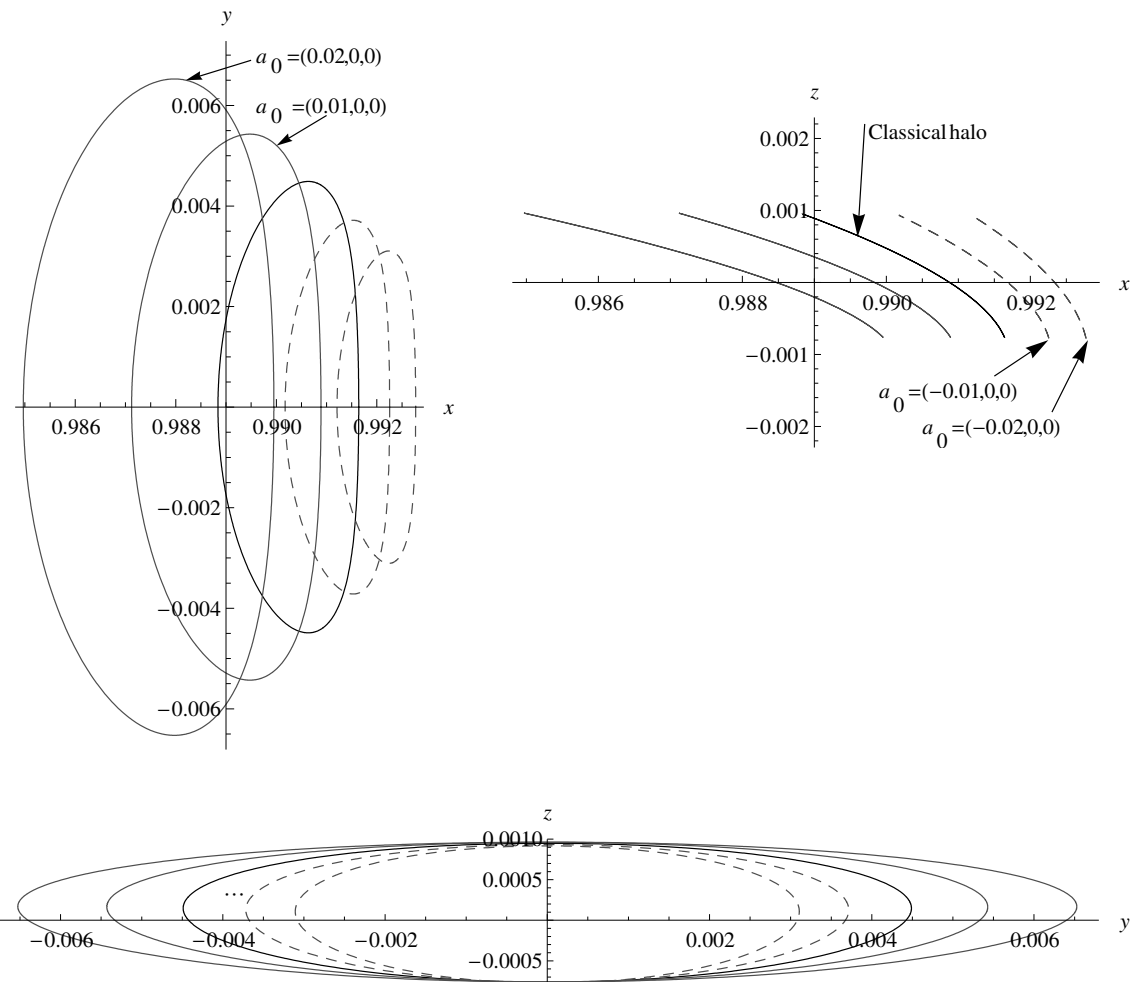

Fig. 7 Artificial halo orbits are shown in gray around artificial $L_{1}$ points $(n=1)$ with low thrust acceleration vectors $\boldsymbol{a}_{0}=( \pm 0.01,0,0)$ and $\boldsymbol{a}_{0}=( \pm 0.02,0,0)$. The classical halo orbit is also shown (3rd dark black orbit). All periodic orbits have same $A_{z}=8.3557 \times$ $10^{-4}(125000 \mathrm{~km}$.

The nonlinear equations of motion Eq. (1) with constant $\boldsymbol{a}_{0}$ are symmetric under the transformation $y \rightarrow-y$ and $t \rightarrow-t$, so this symmetry about the $x z$-plane suggests we need to determine periodic orbits for a half period $T_{1 / 2}$ only. Let $\mathbf{X}_{0}=$ $\left(x_{0}, 0, z_{0}, 0, \dot{y}_{0}, 0\right)$ be initial data from Lindstedt Poincaré, so the spacecraft leaves perpendicularly from the $y=0$ plane. On the first return to the $y=0$ plane, its state is

$$
\mathbf{X}\left(T_{1 / 2}\right)=(\tilde{x}, 0, \tilde{z}, \dot{\tilde{x}}, \dot{\tilde{y}}, \dot{\tilde{z}})
$$

so we have a periodic solution when $\dot{\tilde{x}}=\dot{\tilde{z}}=0$.

Let $\overline{\mathbf{X}}(t)$ represent the reference solution (known) corresponding to $\mathbf{X}_{0}$. This solution can be used to relate the solution of the perturbed initial state $\Delta \mathbf{X}_{0}$ from the reference solution at $t=0$ to its deviation in the final state from the reference solution 

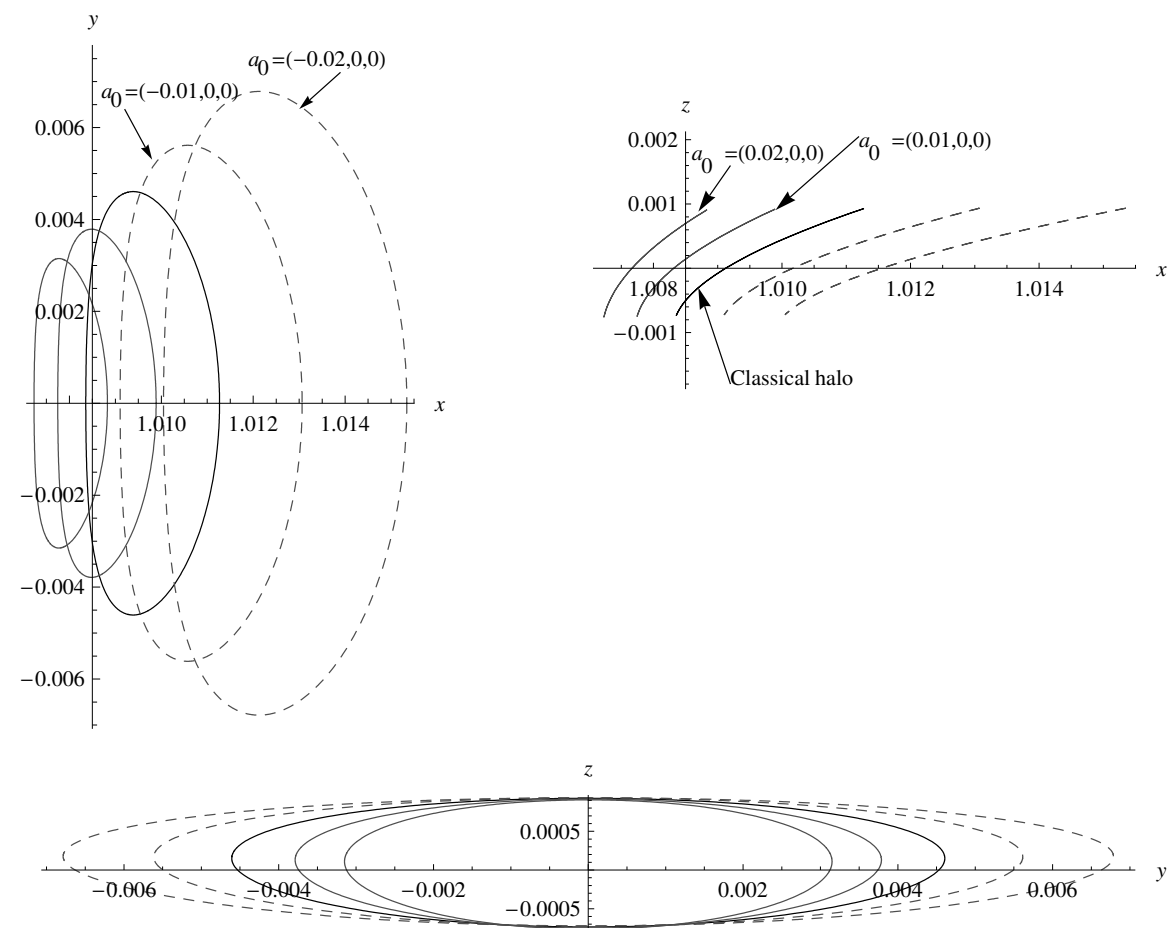

Fig. 8 Artificial halo orbits are shown in gray around artificial $L_{2}$ points $(n=3)$ with low thrust acceleration vectors $\boldsymbol{a}_{0}=( \pm 0.01,0,0)$ and $\boldsymbol{a}_{0}=( \pm 0.02,0,0)$. The classical halo orbit is also shown (3rd dark black orbit). All periodic orbits have same $A_{z}=8.3557 \times$ $10^{-4}(125000 \mathrm{~km}$.)

at $T_{1 / 2}+\Delta T_{1 / 2}$ by

$$
\Delta \mathbf{X}\left(T_{1 / 2}+\Delta T_{1 / 2}\right)=\frac{\partial \mathbf{X}\left(T_{1 / 2}, \mathbf{X}_{0}\right)}{\partial \mathbf{X}_{0}} \Delta \mathbf{X}_{0}+\dot{\mathbf{X}}\left(T_{1 / 2}\right) \Delta T_{1 / 2}
$$

The matrix $\frac{\partial \mathbf{X}}{\partial \mathbf{X}_{0}}=\Phi$ is the state transition matrix evaluated along the reference solution $\overline{\mathbf{X}}(t)$. To make $\dot{\tilde{x}}=\dot{\tilde{z}}=0$ at $y=0$, we vary $x_{0}, \dot{y}_{0}, T_{1 / 2}$ iteratively by corrections $\Delta x_{0}, \Delta \dot{y}_{0}$ and $\Delta T_{1 / 2}$ while keeping $z_{0}$ fixed. These corrections can be calculated from Eq. (13) explicitly as follows

$$
\left(\begin{array}{c}
\Delta x_{0} \\
\Delta \dot{y}_{0} \\
\Delta T_{1 / 2}
\end{array}\right)=\left(\begin{array}{lll}
\phi_{21} & \phi_{25} & \dot{y} \\
\phi_{41} & \phi_{45} & \ddot{x} \\
\phi_{61} & \phi_{65} & \ddot{z}
\end{array}\right)_{t=T_{1 / 2}}^{-1}\left(\begin{array}{c}
0-y \\
0-\dot{\tilde{x}} \\
0-\dot{\tilde{z}}
\end{array}\right)
$$

where $\phi_{i j}$ are elements of the matrix $\Phi$ at $T_{1 / 2}$. 
Figs. (7-8) show numerically generated periodic halo orbits as explained above. The gray orbits are artificial periodic halo orbits around artificial $L_{1}$ points (see Fig. 7) and artificial $L_{2}$ points (see Fig. 8) for low-thrust acceleration values $a_{0}=0.01$ and $a_{0}=0.02$ with the same $A_{z}$. The dashed gray orbits have a low-thrust acceleration vector pointing towards the Sun, so a solar sail cannot generate these periodic artificial halo orbits.

\section{Stable Low-Thrust Halo orbits}

So far we have looked for artificial halo orbits around unstable AEPs. The instability of AEPs implies that artificial halo orbits around these points will also be unstable. However, a continuation method may be used to generate families of periodic orbit with large amplitude and move beyond the region where linear terms dominate so we may find regions of stable halo orbits with low-thrust propulsion.

Given a known periodic solution of Eq. (1) with a known initial condition $\mathbf{X}_{0}$ and parameter of interest (for example $A_{z}$ ), then the continuation method computes the new initial condition to have a periodic orbit for a given fixed new parameter $\left(A_{z}+\Delta A_{z}\right)$. The continuation method, particularly relating to classical halo orbits is discussed in $[4,15]$. Usually the $z-$ amplitude $A_{z}$ is used as a continuation parameter and when it reaches an extreme value, the continuation parameter is changed form $A_{z}$ to $A_{x}$. In this paper, we choose the half period $T_{1 / 2}$ as a continuation parameter. It is found that $T_{1 / 2}$ provides better convergence accuracy than $A_{z}$ and $A_{x}$, the conventional continuation parameters.

For an accurate given periodic orbit $\left(\mathbf{X}_{0}, T_{1 / 2}\right)$, we change the half period from $T_{1 / 2}$ to $T_{1 / 2}^{\prime}=T_{1 / 2}+\Delta T_{1 / 2}$. Then we use $\left(\mathbf{X}_{0}, T_{1 / 2}^{\prime}\right)$ as initial values for integrating Eq. (1) and keep the period fixed at $T_{1 / 2}^{\prime}$. For a fixed period $T_{1 / 2}^{\prime}$, the second term on the right-hand-side of Eq. (13) vanishes, and so the correction in the initial condition $\Delta \mathbf{X}_{0}$ can be calculated as

$$
\Delta \mathbf{X}_{0}=\left.\frac{\partial \mathbf{X}\left(T_{1 / 2}^{\prime}, \mathbf{X}_{0}\right)}{\partial \mathbf{X}_{0}}\right|^{-1} \Delta \mathbf{X}\left(T_{1 / 2}^{\prime}\right)
$$

In particular, to ensure $y, \dot{\tilde{x}}, \dot{\tilde{z}}$ are zero at $T_{1 / 2}^{\prime}$, we are forced to vary $x_{0}, \dot{y}_{0}$ and $z_{0}$ iteratively by corrections $\Delta x_{0}, \Delta \dot{y}_{0}$ and $\Delta z_{0}$. These corrections can be calculated from Eq. (15) as follows

$$
\left(\begin{array}{c}
\Delta x_{0} \\
\Delta \dot{y}_{0} \\
\Delta z_{0}
\end{array}\right)=\left(\begin{array}{lll}
\phi_{21} & \phi_{25} & \phi_{23} \\
\phi_{41} & \phi_{45} & \phi_{43} \\
\phi_{61} & \phi_{65} & \phi_{63}
\end{array}\right)_{t=T_{1 / 2}^{\prime}}^{-1}\left(\begin{array}{c}
0-y \\
0-\dot{\tilde{x}} \\
0-\dot{\tilde{z}}
\end{array}\right)
$$

where $\phi_{i j}$ are elements of the matrix $\Phi$ at $T_{1 / 2}^{\prime}$.

Fig. 9 shows a family of halo orbits about AEPs beyond $L_{2}$. The period corresponding to this family of orbits (thick solid line) is shown in Fig. 10. For comparison, the period of classical halo orbits about $L_{2}$ is also shown. Fig. 11 shows the same family of orbits (see first plot in Fig. 9) characterized by their maximum x-value $x_{\max }$ and maximum z-value $z_{\max }$. Switching from Lindstedt Poincaré analysis at $x_{\max }=1.0126$ to continuation method with $\Delta T_{1 / 2}=-0.02$ causes gaps in the artificial halo orbits curves (see Figs. 10-11). 

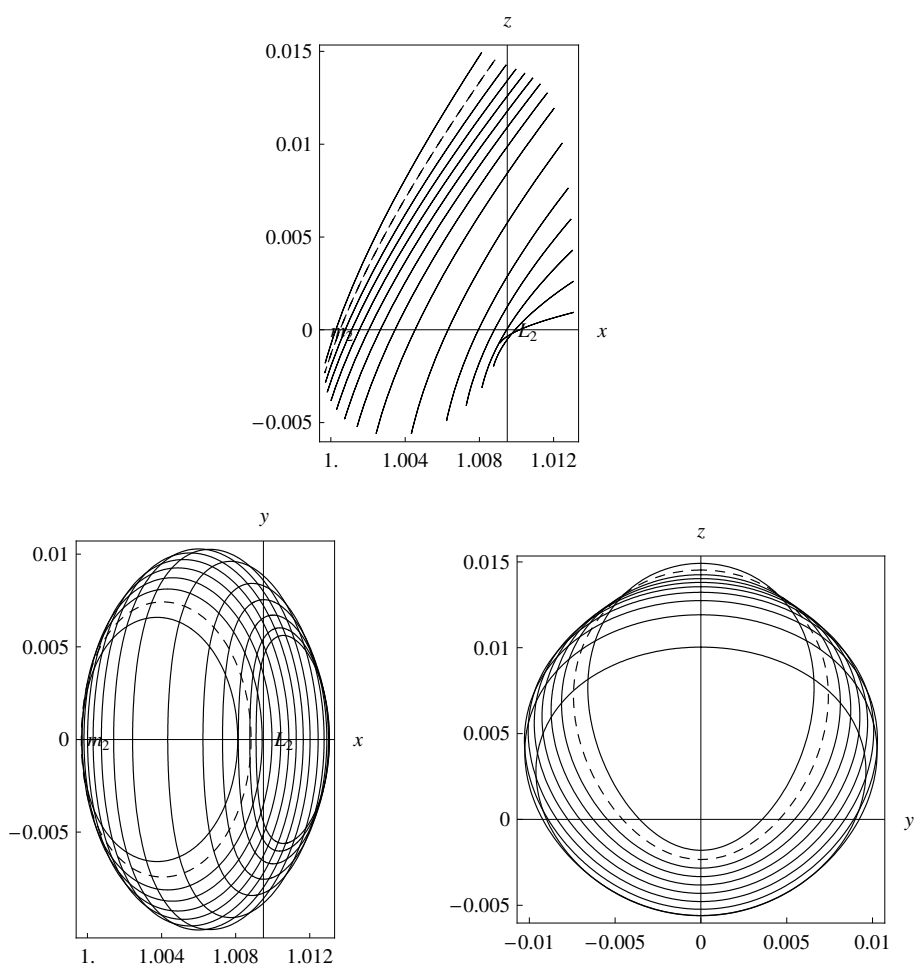

Fig. 9 Artificial periodic halo orbits in the Sun-Earth system around AEP $\mathbf{r}_{0}=(1.01134,0,0)$ with $\boldsymbol{a}_{0}=(-0.01,0,0)$ pointing towards the Sun. The first five periodic orbits are generated by using an initial guess from Lindstedt Poincaré. Large amplitude periodic orbits are produced using the continuation method with $\Delta T_{1 / 2}=-0.02$. The dashed line is the stable halo orbit.

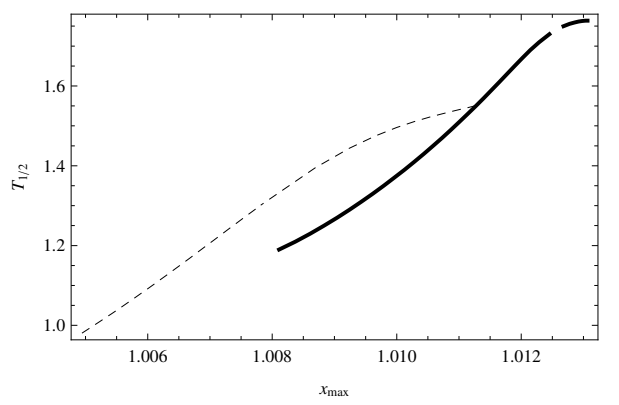

Fig. 10 Half period of classical halo orbits about $L_{2}$ is shown by the dashed line, and the half period of artificial halo orbits about AEP $\mathbf{r}_{0}=(1.01134,0,0)$ is shown by the thick line. 


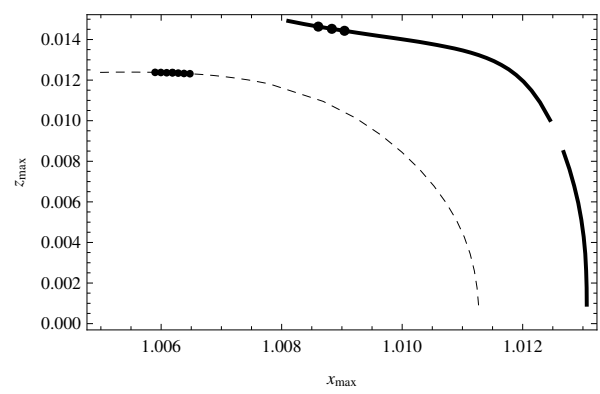

Fig. 11 Classical halo orbits about $L_{2}$ shown by dashed line, and artificial halo orbits about AEP $\mathbf{r}_{0}=(1.01134,0,0)$ with low-thrust acceleration $\boldsymbol{a}_{0}=(-0.01,0.0)$. Heavy dots on both curves corresponds to stable halo orbits.

Table 1 Initial condition for stable orbits with $\boldsymbol{a}_{0}=(-0.01,0,0)$ which corresponds to a low-thrust acceleration $0.0593 \mathrm{mms}^{-2}$ and low-thrust force $30 \mathrm{mN}$ for a $500 \mathrm{~kg}$ spacecraft.

\begin{tabular}{lllll}
\hline$x_{0}\left(\times 10^{8} \mathrm{~km}\right)$ & $z_{0}\left(\times 10^{6} \mathrm{~km}\right)$ & $\dot{y}_{0}\left(\times 10^{2} \mathrm{~m} / \mathrm{s}\right)$ & $T_{1 / 2}($ days $)$ & $C$ \\
\hline 1.509505487164924 & 2.158391940114810 & -4.177777862144087 & 73.8218469 & -1.49000437 \\
1.509191101130694 & 2.173171517835050 & -4.076979879730191 & 72.6592201 & -1.49000439 \\
1.508857610708376 & 2.189749813954399 & -3.966248219678315 & 71.4965932 & -1.49000442 \\
\hline
\end{tabular}

According to Floquet theory, the first order or linear stability of periodic orbits is described by the eigenvalues of the monodromy matrix $\Phi(T)$. Let the nonlinear system Eq. (1) be written as $\dot{X}=\mathbf{f}(\mathbf{X})$. Since the trace of the Jacobian $\frac{\partial \mathbf{f}}{\partial \mathbf{X}}=0$ [see Eq. (5)], eigenvalues of the monodromy matrix occur in reciprocal pairs [16]. The system is autonomous, so it has +1 as an eigenvalue for a periodic orbit[17]. Thus, two of the eigenvalues of the monodromy matrix are unity, and the stability of the periodic orbit is given by the complex conjugate eigenvalues on the unit circle in complex plane. For artificial unstable periodic orbits, the eigenvalues spectrum of the monodromy matrix is given by

$$
\left\{1,1, \lambda_{r}, 1 / \lambda_{r}, \lambda_{i}, \bar{\lambda}_{i}\right\}
$$

For stable periodic orbits, the spectrum of the monodromy matrix is described by

$$
\left\{1,1, \lambda_{i}, \bar{\lambda}_{i}, \lambda_{j}, \bar{\lambda}_{j}\right\}
$$

i.e., all eigenvalues lies on the unit circle, and the periodic orbit keeps a halo shape for large integration times. The initial condition for such stable artificial periodic orbits is given in Table 1. In the Sun-Earth system, the low-thrust acceleration $0.0593 \mathrm{~mm} / \mathrm{s}^{2}$ corresponds to $30 \mathrm{mN}$ thrust for a $500 \mathrm{~kg}$ spacecraft. Assuming $I_{s p}=3200 \mathrm{~s}$ and propellant mass percentage of $50 \%$, the propellant will be consumed within 11.5 years. However, these stable orbits can keep a halo shape for larger integration times i.e, 25 years. Stable halo orbits (shown by dots in Fig. 11) about $L_{2}$ are between the $L_{2}$ and the Earth, while artificial stable orbits in the case of low-thrust propulsion about AEP $\mathbf{r}_{0}=(1.01134,0,0)$ are closer to $L_{2}$. Although the existence of stable halo orbits for low-thrust propulsion spacecraft is shown around unstable AEP, the in-depth stability analysis could be made $[18,19]$, and is left to future work. 


\section{Conclusions}

We have shown the possibility of generating halo orbits, using near-term electric propulsion system in the circular restricted three body problem around nonequilibrium points by changing these points into equilibrium points with low-thrust acceleration. In particular halo orbits around nonequilibrium points inside $L_{1}$ and beyond $L_{2}$ that require the low-thrust acceleration directed towards sunward are shown to be feasible with such low-thrust propulsion. It is therefore impossible for solar sails, to generate these artificial halo orbits. We have also shown that we may fine tune the initial data provided by the Lindstedt Poincaré method, for integration of nonlinear equations of motion with constant continuous low-thrust acceleration, to produce closed orbits around artificial equilibrium point using a differential corrector. Stable low-thrust halo orbits for a point beyond $L_{2}$ are also found using a continuation method, while the continuing parameter is chosen as the half period of the halo orbit. These stable halo orbits are realizable with solar electric propulsion and found to be towards $L_{2}$, while the classical stable halo orbits around $L_{2}$ are roughly halfway between the Earth and $L_{2}$.

\section{Acknowledgments}

The first author acknowledgments useful discussions with Dr. Thomas J. Waters and Dr. James D. Biggs.

\section{References}

1. Farquhar, R. and Kamel, A., "Quasi-periodic orbits about the trans-lunar libration point," Celestial Mechanics, Vol. 7, 1973, pp. 458-473.

2. Breakwell, J. and Brown, J., "The 'halo' family of 3-dimensional periodic orbits in the Earth-Moon restricted 3-body problem," Celestial Mechanics, Vol. 20, 1979, pp. 389-404.

3. Richardson, D. L., "Halo orbit formulation for the ISEE-3 mission," J. Guidance and Control, Vol. 3, No. 6, 1980, pp. 543-548.

4. Howell, K., "Three-dimensional, periodic, 'halo' orbits," Celestial Mechanics, Vol. 32, 1984, pp. 53-71.

5. Thurman, R. and Worfolk, P., "The geometry of halo orbits in the circular restricted threebody problem," Technical report GCG95, Geometry Center, University of Minnesota, 1996.

6. Richardson, D. L., "Analytical construction of periodic orbits about the collinear points," Celestial Mechanics and Dynamical Astronomy, Vol. 22, No. 3, 1980, pp. 241-253.

7. McInnes, C., McDonald, A., Simmons, J., and McDonald, E., "Solar sail parking in restricted three-body systems," Journal of Guidance, Control and Dynamics, Vol. 17, No. 2, 1994, pp. 399-406.

8. McInnes, A., "Strategies for solar sail mission design in the circular restricted three-body problem," Masters Thesis, Purdue University, 2000.

9. Baoyin, H. and McInnes, C., "Solar sail halo orbits at the Sun-Earth artificial L 1 point," Celestial Mechanics and Dynamical Astronomy, , No. 94, 2006, pp. 155-171.

10. Waters, T. and McInnes, C., "Periodic orbits above the ecliptic in the solar sail restricted 3-body problem," Journal of Guidance, Control and Dynamics, Vol. 30, No. 3, 2007, pp. $687-693$.

11. Morimoto, M., Yamakawa, H., and Uesugi, K., "Artificial Equlibrium Points in the LowThrust Restricted Three-Body Problem," Journal of Guidance, Control and Dynamics, Vol. 30, No. 5, 2007, pp. 1563-1567.

12. Morimoto, M., Yamakawa, H., and Uesugi, K., "Periodic orbits with Low-Thrust Propulsion in the Restricted Three-Body Problem," Journal of Guidance, Control and Dynamics, Vol. 29, No. 5, 2006, pp. 1131-1139. 
13. Betounes, D., Differential Equations: theory and applications, Springer-Verlag, New York, 2001, pp. 200-205.

14. Battin, R. H., An Introduction to the Mathematics and Methods of Astrodynamics, AIAA, Reston, VA, rev. ed., 1999, pp. 573.

15. Kim, M. and Hall, C., "Lyapunov and Halo Orbits about $L_{2}, "$ AAS/AIAA Astrodynamics Specialist Conference, Quebec City, Canada, AAS 01-324, Jul 30-Aug 2, 2001.

16. Broucke, R., Lass, H., and Boggs, D., "A note on the solution of the variational equations of a class of dynamical systems," Celestial Mechanics and Dynamical Astronomy, Vol. 14, No. 3, 1976, pp. 383-392.

17. Whittaker, E., A treatise on the analytical dynamics of particles and rigid bodies, Cambrige University Press, New York, 4th ed., 1999, pp. 398-399.

18. Lara, M., Russel, R., and Villac, B., "Fast estimation of Stable Regions in Real Mode," Celestial Mechanics and Dynamical Astronomy, Vol. 42, No. 5, October 2007, pp. 511-515.

19. Villac, B. F., "Using FLI Maps for Preliminary Spacecraft Trajectory Design in MultiBody Environments," Celestial Mechanics and Dynamical Astronomy, Vol. 102, No. 1-3, September 2008, pp. 29-48. 